\title{
On the Kinematic Design of Exoskeletons and Their Fixations with a Human Member
}

\author{
Nathanaël Jarrassé \\ Guillaume Morel \\ University P. \& M. Curie, ISIR (Institut des Systèmes Intelligents et de Robotique) CNRS - UMR 7222 \\ 4 place Jussieu, 75005 Paris - France \\ Emails : jarrasse@isir.fr, guillaume.morel@upmc.fr
}

\begin{abstract}
A crucial problem in developing robotic exoskeletons lies in the design of physical connexions between the device and the human limb it is connected to. Indeed, because in general the human limb kinematics and the exoskeleton kinematics differ, using an embedment at each connection point leads to hyperstaticity. Therefore, uncontrollable forces can appear at the interaction port. To cope with this problem, literature suggests to add passive mechanisms at the fixation points. However, empirical solutions proposed so far suffer from a lack of proper analysis and generality.

In this paper, we study the general problem of connecting two similar kinematic chains through multiple passive mechanisms. We derive a constructive method that allows to determine all the possible repartitions of freed DoFs across the different fixation mechanisms. It also provides formal proofs of global isostaticity. Practical usefulness is illustrated through an example with conclusive experimental results.
\end{abstract}

\section{INTRODUCTION}

More and more exoskeletons are being designed by researchers for a growing number of applications, ranging from military applications [1] to rehabilitation [2].

For years, research has mainly focused on technological aspects (actuators, embedment, energy...) and followed a paradigm defined in [3]: "an exoskeleton is an external structural mechanism with joints and links corresponding to those of the human body" . In other words, designing the kinematics of an exoskeleton generally consists of trying to replicate the human limb kinematics. This brings a number of advantages: similarity of the workspaces, singularity avoidance [4], oneto-one mapping of joint force capabilities over the workspace. The major drawback of this paradigm is that, in fact, human kinematics is impossible to precisely replicate with a robot. Indeed two problems occur: morphology drastically varies between subjects and, for a given subject, the joints kinematics is very complex and cannot be imitated by conventional robot joints [5]. In fact, it is impossible to find any consensual model of the human kinematics in the biomechanics literature due to complex geometry of bones interacting surfaces. For example, different models are used for the shoulder-scapulaclavicle group [6].

Discrepancies between the two kinematic chains thus seem unavoidable. Because of the connexions between multiple loops, it generates kinematic compatibility problems. Indeed, when connecting two-by-two the links of two kinematically similar chains that are not perfectly identical, hyperstaticity occurs. This phenomenon leads, if rigid models are used, to the impossibility of moving and the appearance of noncontrollable (possibly infinite) internal forces. In practice, though, rigidity is not infinite and mobility can be obtained thanks to deformations. When a robotic exoskeleton and a human limb are connected, most likely, these deformations occur at the interface between the two kinematic chains, caused by the low stiffness of human skin and tissues surrounding the bones [7].

Solutions found in the literature to cope with this problem are of two kinds. In a first approach the exoskeleton design can be thought in such a way that adaptation to human limb kinematics is maximized. Robotic segments with adjustable length were thus developed, and pneumatic systems were added to introduce elasticity in the robot fixations and adaptability to variable limb section [8]. This minimizes the kinematic differences, but drastically increases the complexity of the device, leading to weight increase, stiffness limitations, etc. Furthermore, again, it seems that perfect matching at any instant is yet out of reach.

The second approach consists in adding passive DoFs to connect the two kinematic chains. This was proposed back in the 1970s in the context of passive orthoses, [9], [10]. The same principle was recently proposed for a one degree of freedom device in [7], but the force transmission is analyzed only in a plane, and relies on explicit equations derived for a particular planar mechanism. It thus suffers from a lack of generality and the author neglects all the off-plane forces that unavoidably arise from the unmodeled lack of parallelism between the human limb plane and the exoskeleton plane.

Rather, the constructive method proposed here applies to a general spatial problem, which is properly formalized and then solved thanks to a set of necessary and sufficient conditions for global isostaticity (Section II). In Section III, the method is applied to ABLE, a given active 4DoF arm exoskeleton. In Section IV, experimental results illustrate the practical interest of the approach.

\section{General MEthodology}

The main question addressed in this paper is: given a proposed exoskeleton structure designed to (approximately) 
replicate a human limb kinematic model, how to connect it to the human limb while avoiding the appearance of uncontrollable forces at the interface? The answer takes the form of a set of passive frictionless mechanisms used to connect the robot and the subject's limb that allows to avoid hyperstaticity.

\section{A. Problem formulation}

We consider two different serial chains with multiple couplings as illustrated in Fig. 1. One represents a human limb $\mathbf{H}$ and the other the robot structure $\mathbf{R}$.

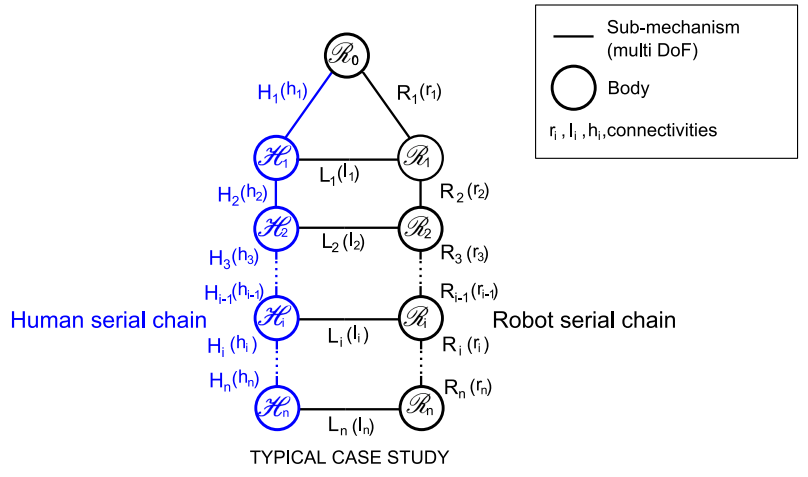

Fig. 1. Schematic of two serial chains parallel coupling

The base body of the exoskeleton is supposed to be attached to a body of the human subject. This common body is denoted $\mathscr{R}_{0} \equiv \mathscr{H}_{0}$. The robot and the limbs are supposed to be connected through $n$ fixations. Each fixation is a mechanism $\mathbf{L}_{i}$ for $i \in\{1, . ., n\}$ consisting in a passive kinematic chain which connects a human body $\mathscr{H}_{i}$ to a robot body $\mathscr{R}_{i}$. Mechanisms $\mathbf{L}_{i}$ are supposed to possess a connectivity $l_{i}$. Recall that connectivity is the minimum and necessary number of joint scalar variables that determine the geometric configuration of the $\mathbf{L}_{i}$ chain [11]. Typically, $\mathbf{L}_{i}$ will be a nonsingular serial combination of $l_{i}$ one DoF joints. The fixation can be an embedment $\left(l_{i}=0\right)$ or can release several DoFs, such that:

$$
\forall i \in\{1, . ., n\}, \quad 0 \leq l_{i} \leq 5 .
$$

Indeed choosing $l_{i} \geq 6$ would correspond to complete freedom between $\mathscr{H}_{i}$ and $\mathscr{R}_{i}$ which would not make any practical sense in the considered application where force transmission is required.

Between $\mathscr{R}_{i-1}$ and $\mathscr{R}_{i}$, on the robot side, there is an active mechanism $\mathbf{R}_{i}$ which connectivity is denoted $r_{i}$. Similarly, between $\mathscr{H}_{i-1}$ and $\mathscr{H}_{i}$ on the human side, there is a mechanism $\mathbf{H}_{i}$ of connectivity $h_{i}$. Note that, due to the complexity of human kinematic $h_{i}$ is not always exactly known, and literature from biomechanics provides controversial data on this point. For example, the elbow is often modeled as a one DoF joint, but in reality a residual second DoF can be observed [12].

Our goal is to design mechanisms $\mathbf{L}_{i}$ with $i \in\{1, . ., n\}$ in such a way that on one side, all the forces generated by the exoskeleton on the human limb are controllable and on the other side, there is no possible motion for the exoskeleton when the human limb is still. We shall thus consider in the next that the human limbs are virtually attached to the base body $\mathscr{R}_{0}$. This represents the case, when the subject does not move at all. The resulting mechanism, depicted in Fig. 2, is denoted $S_{n}$.

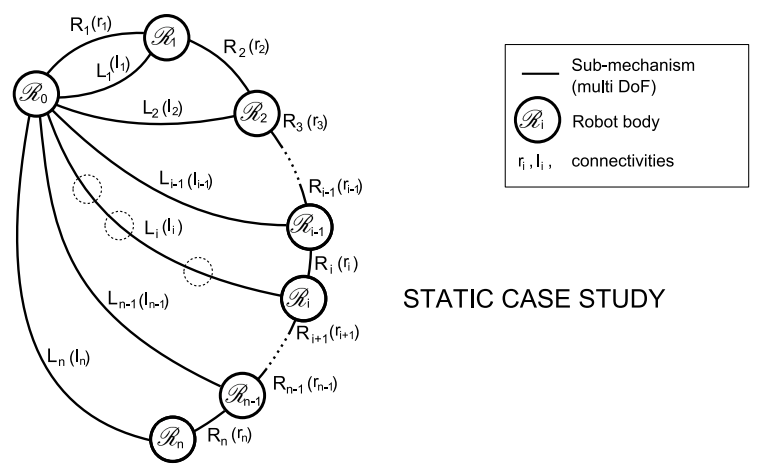

Fig. 2. Studied problem with a fixed human limb

A proper design for the passive mechanisms $\mathbf{L}_{i}$ shall guarantee that, in the absence of any external forces, both:

$$
\begin{aligned}
& \forall i \in 1 \cdots n, \quad{ }^{\mathbf{S}_{n}} T_{i}=\{0\} \quad \text { and } \\
& \forall i \in 1 \cdots n, \quad{ }^{\mathbf{S}_{n}} W_{\mathbf{L}_{i} \rightarrow 0}=\{0\},
\end{aligned}
$$

where ${ }^{S_{n}} T_{i}$ is the space of twists describing the velocities of robot body $\mathscr{R}_{i}$ relative to $\mathscr{R}_{0}$ when the whole mechanism $\mathbf{S}_{n}$ is considered and ${ }^{\mathbf{S}_{n}} W_{\mathbf{L}_{i} \rightarrow 0}$ is the space of wrenches (forces and moments) statically admissible transmitted through the $\mathbf{L}_{i}$ chain on the reference body $\mathscr{R}_{0}$, when the whole mechanism $\mathbf{S}_{n}$ is considered.

Equation (2a) expresses the fact that the mobility of any robot body connected to a human limb should be null, which is required since the human member is supposed here to be still. Moreover, Eq. (2b) imposes that, considering the whole mechanism, there can be no forces of any kind exerted on the human limb. Indeed, since the actuators are supposed to apply null generalized forces, the presence of any force at the connection ports would be an uncontrollable force due to hyperstaticity. In the next Eq. (2) is referred as the global isostaticity condition.

\section{B. Conditions on the twist space ranks}

At first, one can notice the recursive structure of the considered system: if we name $\mathbf{S}_{i}$ the sub-mechanism constituted by the bodies $\mathscr{R}_{0}$ to $\mathscr{R}_{i}$, the chains $\mathbf{R}_{0}$ to $\mathbf{R}_{i}$ and $\mathbf{L}_{0}$ to $\mathbf{L}_{i}$, we can represent $\mathbf{S}_{i}$ recursively from $\mathbf{S}_{i-1}$, as in Fig. 3, where $m_{i-1}$

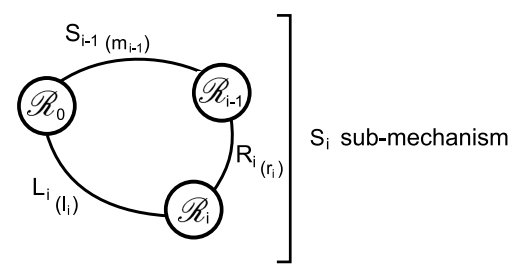

Fig. 3. Recursive structure $\mathbf{S}_{i}$ of the system

is the connectivity of $\mathbf{S}_{i-1}$. In this convention, $\mathbf{S}_{0}$ represents a 
zero DoF mechanism. Using this recursive representation one can establish the following proposition:

Proposition 1: The conditions (2) are equivalent to :

$$
\begin{array}{cc}
\forall i \in 1 \cdots n, \quad \operatorname{dim}\left(T_{\mathbf{S}_{i-1}}+T_{\mathbf{R}_{i}}+T_{\mathbf{L}_{i}}\right)=6 & \text { and } \\
\forall i \in 1 \cdots n, \quad \operatorname{dim}\left(T_{\mathbf{S}_{i-1}} \cap T_{\mathbf{R}_{i}}\right)=0 & \text { and } \\
\operatorname{dim}\left(T_{\mathbf{S}_{n}}\right)=0, &
\end{array}
$$

where $T_{\mathbf{S}_{j}}=\mathbf{S}_{j} T_{j}$ is the space of twists describing the velocities of $\mathscr{R}_{j}$ relative to $\mathscr{R}_{0}$, when $\mathbf{S}_{j}$ is considered isolated from the rest of the mechanism (then it is different from ${ }^{\mathbf{S}_{n}} T_{j}$ ), $T_{\mathbf{R}_{i}}$ is the space of twists produced by $\mathbf{R}_{i}-i$.e. the space of twists of $\mathscr{R}_{i}$ relative to $\mathscr{R}_{i-1}$ if they were only connected through $\mathbf{R}_{i}, T_{\mathbf{L}_{i}}$ is the space of twists produced by $\mathbf{L}_{i}$ i.e. the space of twists of $\mathscr{R}_{i}$ relative to $\mathscr{R}_{0}$ if they were only connected through $\mathbf{L}_{i}$.

The demonstration can be found in Appendix A.

Remarkably, conditions (3) involve the space of twists generated by $\mathbf{R}_{i}$ and $\mathbf{L}_{i}$ when taken isolated, which is of great help for design purposes. In the next, we convert these conditions into constraints on the connectivities $r_{i}=\operatorname{dim}\left(T_{\mathbf{R}_{i}}\right)$ and $l_{i}=$ $\operatorname{dim}\left(T_{\mathbf{L}_{i}}\right)$. To do so, we suppose that kinematic singularities are avoided. In other words, summing the subspaces of twists will always lead to a subspace of maximum dimension given the dimensions of individual summed subspaces. This hypothesis will lead to determine how many DoFs shall be included in the passive fixation mechanisms $\mathbf{L}_{i}$. Of course as it is usual in mechanism design, when a particular design is finally proposed, it will be necessary to verify a posteriori the singularity avoidance condition.

\section{Conditions on connectivities}

At first, let's compute the connectivity of $\mathbf{S}_{i}$. One has:

$$
T_{\mathbf{S}_{i}}=T_{\mathbf{L}_{i}} \cap\left(T_{\mathbf{R}_{i}}+T_{\mathbf{S}_{i-1}}\right),
$$

which directly results from the space sum law for serial chains and the intersection law for parallel chains (see [13]). Furthermore, since for any vector subspaces $\mathbf{A}$ and $\mathbf{B}, \operatorname{dim}(\mathbf{A})+$ $\operatorname{dim}(\mathbf{B})=\operatorname{dim}(\mathbf{A}+\mathbf{B})+\operatorname{dim}(\mathbf{A} \cap \mathbf{B})$, one gets:

$$
\begin{aligned}
m_{i}= & \operatorname{dim}\left(T_{\mathbf{L}_{i}}\right)+\operatorname{dim}\left(T_{\mathbf{R}_{i}}+T_{\mathbf{S}_{i-1}}\right)-\operatorname{dim}\left(T_{\mathbf{L}_{i}}+T_{\mathbf{R}_{i}}+T_{\mathbf{S}_{i-1}}\right) \\
= & \operatorname{dim}\left(T_{\mathbf{L}_{i}}\right)+\operatorname{dim}\left(T_{\mathbf{R}_{i}}\right)+\operatorname{dim}\left(T_{\mathbf{S}_{i-1}}\right)-\operatorname{dim}\left(T_{\mathbf{R}_{i}} \cap T_{\mathbf{S}_{i-1}}\right) \\
& -\operatorname{dim}\left(T_{\mathbf{L}_{i}}+T_{\mathbf{R}_{i}}+T_{\mathbf{S}_{i-1}}\right) .
\end{aligned}
$$

If condition (3) is respected and under full rank asumption, one gets:

$$
m_{i}=l_{i}+r_{i}+m_{i-1}-6
$$

Finally, using $m_{0}=0$, this recursive equation simplifies to:

$$
m_{i}=\sum_{j=1}^{i}\left(l_{j}+r_{j}\right)-6 . i
$$

Therefore, from Eq. (3a), noticing that any vector subspaces $\mathbf{A}, \mathbf{B}$ and $\mathbf{C}$ of a vector space $\mathbf{E}, \operatorname{dim}(\mathbf{A}+\mathbf{B}+\mathbf{C}) \leq \operatorname{dim}(\mathbf{A})+$ $\operatorname{dim}(\mathbf{B})+\operatorname{dim}(\mathbf{C})$, it is necessary that:

$$
\forall i \in 1 \cdots n, \quad m_{i-1}+r_{i}+l_{i} \geq 6, \quad \text { or }: \quad \sum_{j=1}^{i}\left(l_{j}+r_{j}\right) \geq 6 . i
$$

Moreover, since if $\mathbf{A}$ and $\mathbf{B}$ are two vector subspaces of $\mathbf{E}$ and $\operatorname{dim}(\mathbf{A})+\operatorname{dim}(\mathbf{B})>\operatorname{dim}(\mathbf{E})$, then $\mathbf{A} \cap \mathbf{B} \neq\{0\}$, Eq. (3b) imposes that:

$$
\forall i \in 1 \cdots n, \quad m_{i-1}+r_{i} \leq 6 \text { or }: \sum_{j=1}^{i-1}\left(l_{j}+r_{j}\right)+r_{i} \leq 6 . i
$$

Finally, the last condition (3c) leads to:

$$
m_{n}=0 \text { or }: \sum_{j=1}^{n}\left(l_{j}+r_{j}\right)=6 . n
$$

Notice that (9) provides the total number of DoFs to be freed for the mechanism $\mathbf{S}_{n}$, while (7) gives the minimal value (to prevent from hyperstaticity in the sub-mechanisms $\mathbf{S}_{j}$ ) for $l_{j}$ and (8) provides the maximal one (to prevent from internal mobility in $\mathbf{S}_{j}$ ).

Thanks to these three last necessary conditions, we are able to calculate the different possible solutions for distributing the additional passive DoFs over the structure:

- the possible choices for $l_{1}$ are such that $5 \geq l_{1} \geq 6-r_{1}$.

- for each choice of $l_{1}$, the possible choices for $l_{2}$ are such that $5 \geq l_{2} \geq 12-r_{1}-r_{2}-l_{1}$.

This leads to a tree that groups all the admissible combinations for $l_{i}$, as illustrated in Fig (4).

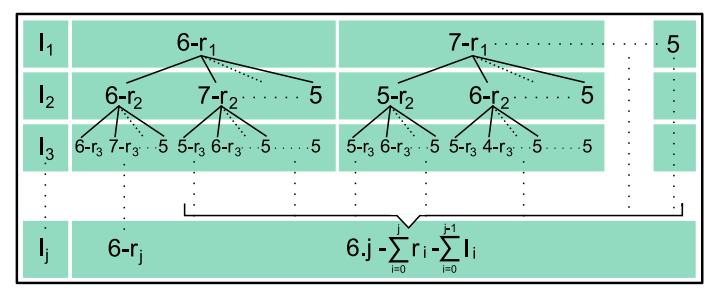

Fig. 4. Tree of possible solutions for the number of passive DoFs to add at every fixation point

Out of this tree, all the possible combinations of connectivities for the fixations are given. Of course, the selection among these solutions is to be made depending on the exoskeleton kinematics. Generally speaking, an important aspect to be considered is the force transmission: through any linear or rotational DoF that is not freed by the fixation mechanism, a force or a moment will be transmitted to the human limb, which is surrounded by soft tissues. Therefore, typically, transmitting moments around $P_{i}$ would lead to locally deform the tissues which in turn can generate discomfort. The next section illustrates, on a concrete spatial example involving two fixations, how to integrate this kind of considerations in the design of fixation mechanisms.

\section{ApPlicAtion to A GIVEN EXOSKELETON}

\section{A. ABLE: an upper limb exoskeleton for rehabilitation}

ABLE (see Fig. 5) is a 4 axis exoskeleton that has been designed by CEA-LIST [14] on the basis of an innovative screw-and-cable actuation technology ([15]). Its kinematics is composed of a shoulder spherical joint composed of 3 coincident pivots and a 1 DoF pivot elbow. The forearm, 


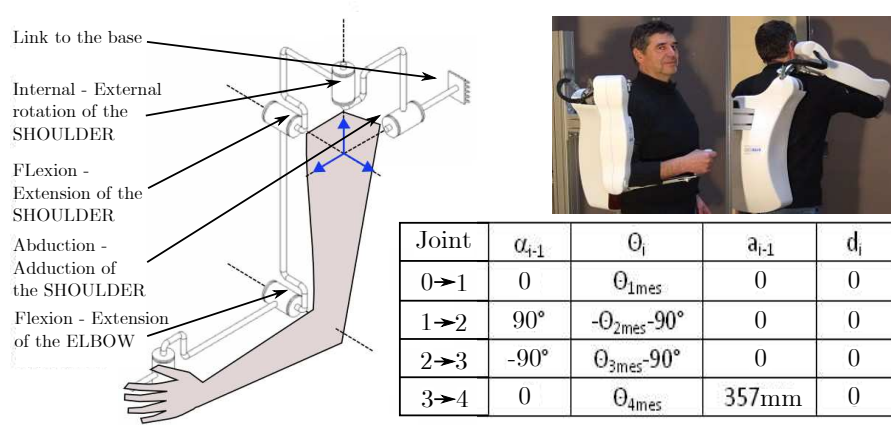

Fig. 5. Kinematics of ABLE

terminated by a handle, is not actuated. Details on this robot can be found in [14].

\section{B. Fixations design for $A B L E$}

In this section, we apply general method proposed in Sec. II to ABLE. We proceed in three steps:
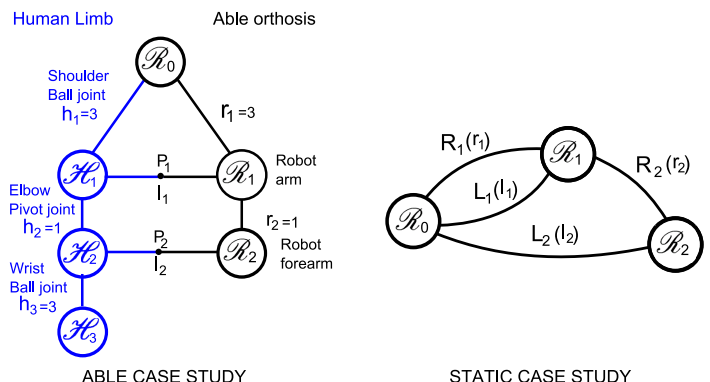

Fig. 6. Schematic of the ABLE and human arm coupling

- compute the tree of possible values for $l_{i}$

- choose among them a preferred solution by examining force transmission properties and kinematic complementarity

- verify the full kinematic rank which is reported in Appendix B.

Firstly, since ABLE comprises an upper arm and a forearm, we shall use two fixations (See Fig 6). The total number of passive DoF to be added is given by Eq. (9):

$$
\sum_{j=1}^{n=2} l_{j}=12-\sum_{j=1}^{n=2} r_{j}=12-(3+1) \quad \Rightarrow l_{1}+l_{2}=8
$$

Moreover, for the first fixation, Eq. (7) and (8) give:

$$
6-r_{1} \leq l_{1} \leq 6 \quad \Rightarrow \quad 3 \leq l_{1} \leq 5 .
$$

Since the total number of DoFs is fixed, the tree of possible solutions consists here of three parallel branches where $l_{1}$ is chosen between 3 and 5 and $l_{2}=8-l_{1}$. Possible couples for $\left(l_{1}, l_{2}\right)$ are $(3,5),(4,4)$ and $(5,3)$. Hereafter, these three options are analyzed in order to choose a preferred design among them.

- Case $a: l_{1}=3$ and $l_{2}=5$. In this case, both $\mathbf{S}_{1}$ taken isolate and $\mathbf{S}_{2}$ are isostatic, which corresponds to the most intuitive way of achieving global isostaticity. Degrees of Freedom for $\mathbf{L}_{1}$ have to be chosen complementary to those of $\mathbf{R}_{1}$ in order to satisfy the full rank assumption. Since $\mathbf{R}_{1}$ is a ball joint that generates three independent rotational velocities around its center $M_{1}, \mathbf{L}_{1}$ must generate three independent velocities at point $M_{1}$. For example, three non coplanar translations could be used for $\mathbf{L}_{1}$. However, in this case, the fixation would transmit a null force, i.e. a pure couple. This seems undesirable due to the torsion of the soft tissues that it would create around $P_{1}$ at the level of the attachment to the limb. One could thus think of using for $\mathbf{L}_{1}$ a ball joint around $P_{1}$, but in this case, the full rank condition would not be respected, because $\mathbf{R}_{1}$ and $\mathbf{L}_{1}$ would both generate the same rotation around $\vec{z}_{1}=\frac{1}{\left\|\overrightarrow{M_{1} P_{1}}\right\|} \overrightarrow{M_{1} P_{1}}$. Finally, a preferred solution is to choose for $\mathbf{L}_{1}$ two pivot joints perpendicular to the arm main axis $\vec{z}_{\text {arm }}$, and one translation joint collinear $\vec{z}_{\text {arm }}$ (see 8 for further definition of $P_{i}, \vec{z}_{\text {arm }}$ and $\vec{z}_{\text {forearm }}$ ). In this case, two forces perpendicular to $\vec{z}_{\text {arm }}$ and one moment around $\vec{z}_{\text {arm }}$ can be exchanged between the exoskeleton and the arm through $\mathbf{L}_{1}$. Moreover, since $\mathbf{S}_{1}$ is isostatic, one has $m_{1}=0$. Therefore $\mathbf{L}_{2}$ needs to be designed in order to be kinematically complementary to $\mathbf{R}_{2}$, which is a pivot of axis $\left(M_{2}, \vec{z}_{2}\right)$ (See Appendix for $\vec{z}_{2}$ definition). In other words, $\mathbf{L}_{2}$ must generate independently 2 rotations perpendicular to $\vec{z}_{2}$ and 3 velocities at point $M_{2}$. A natural solution is to choose a ball joint around $P_{2}$ and two translations in a plane perpendicular to $\vec{z}_{2}$. The resulting overall design is noted (a) and represented in Fig. 7.
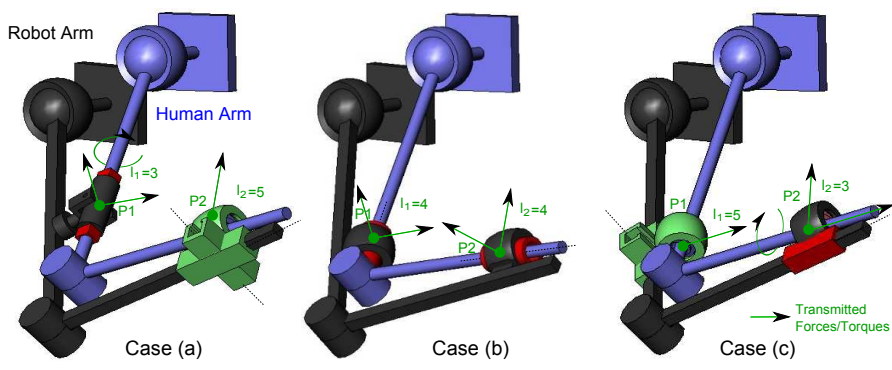

Fig. 7. Considered possibilities for coupling ABLE to an human arm. Case (a): ball joint alone at $P_{1}$ and ball joint +2 slides at $P_{2}$; case (b): Universal joint +1 slide at $P_{1}$ and ball joint +2 slides at $P_{2}$; case (c) Ball joints with slide at both $P_{1}$ and $P_{2}$.

- Case b: $l_{1}=4$ and $l_{2}=4$. Note that in this case, $\mathbf{S}_{1}$ taken isolate is a 1 DoF mechanism, while only $\mathbf{S}_{2}$ is isostatic. We consider solution (a), for which one DoF must be added to $\mathbf{L}_{1}$ and one must be removed from $\mathbf{L}_{2}$. Concerning $\mathbf{L}_{1}$, keeping freed the 3 DoF liberated for the isostatic solution (a), it seems preferable to choose, for the extra freed DoF, the rotation around $z_{1}$. Indeed, this will cancel the local tissue torsion due to moment transmission around $\vec{z}_{1}$. As a result, $\mathbf{S}_{1}$ is now a 1 DoF mechanism consisting of a pivot around $\left(M_{1}, \vec{z}_{1}\right)$. Concerning $\mathbf{L}_{2}$, the DoF to be removed from the solution (a) shall not degrade the dimension of $T_{\mathbf{S}_{1}}+T_{\mathbf{R}_{2}}+T_{\mathbf{L}_{2}}$. It seems preferable to keep the freed three rotations around $P_{2}$ and only one translation along the forearm axis $\vec{z}_{\text {forearm }}$. Indeed, again, this choice avoids any torsion around $P_{2}$. Furthermore, it is 
shown in Appendix B that singular configurations of this solution, noted (b) and represented in Fig. 7 are easily identifiable and far away from nominal conditions of operation.

- Case c: $l_{1}=5$ and $l_{2}=3$. Similarly to solution (a), this combination will necessary lead to transmit at least one torsion moment around $\vec{z}_{\text {forearm }}$, as illustrated in Fig. 7 (solution (c)). Therefore, the finally preferred solution is (b).

Note that with solution (b), generating a moment to the human upper arm around $\vec{z}_{\text {arm }}$ is obtained by applying opposite pure forces perpendicular to $\vec{z}_{\text {arm }}$ at $P_{1}$ and to $\vec{z}_{\text {forearm }}$ at $P_{2}$ (see

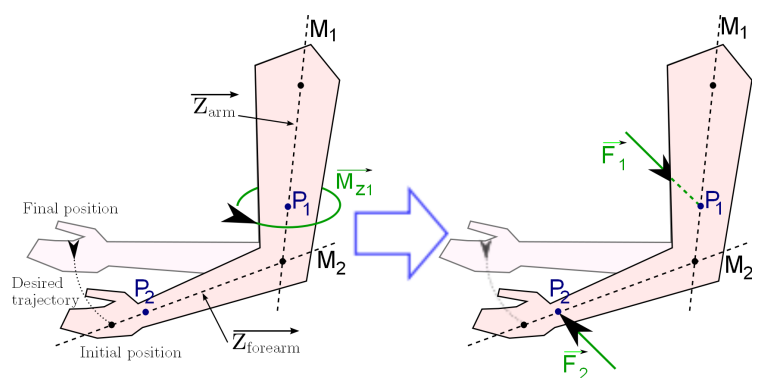

Fig. 8. Transmitting a moment around the upper arm axis with solution (b) (left) and (c) (right)

Fig. 8). Interestingly, this reproduces the method used by physical therapists to assist patients in generating internal rotations of the shoulder without torsion to the tissue. As a price, the full extension configuration, when $M_{1}, P_{1}$ and $P_{2}$ are aligned, is singular, as detailed in the Appendix B. This configuration can be easily avoided by limiting the range of the elbow extension.

\section{Fixations realization}

The two fixations mechanisms are finally identical. They shall generate three independent rotations and one translation along the limb. The mechanism used to realize this function

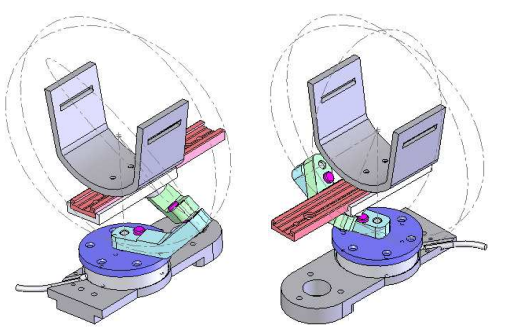

Fig. 9. Fixation simplification and realization (rear and front)

consists of three successive pivot joints which axis coincide and one slider whose axis is parrallel to human limb (see Fig 9).

The fixations were dimensionned differently: one to allow forearm pronosupination and the other not to collide with arm tissues. As a result, possible motions left by the passive fixations have the ranges defined in Table I.

These fixations were both fitted with one force sensor placed on the base (ATI Nano43 6-axis Force/Torque sensor) allowing us to reconstruct the three forces and three torques components

\begin{tabular}{|l|c|c|}
\hline DoF & Arm Fixation & Forearm Fixation \\
\hline Rotation1 ( $\perp$ to the limb axis) & $360^{\circ}$ & $360^{\circ}$ \\
\hline Rotation2 $(\perp$ to the limb axis) & $90^{\circ}$ & $90^{\circ}$ \\
\hline Rotation3 (around the limb axis) & $110^{\circ}$ & $110^{\circ}$ \\
\hline Translation & $100 \mathrm{~mm}$ & $100 \mathrm{~mm}$ \\
\hline
\end{tabular}

TABLE I

at $P_{1}$ and $P_{2}$ respectively.

For the experiments presented in the next section, in order to compare the forces involved with and without DoF liberation, the fixations were also equipped with removable metallic pins, allowing us to quickly lock the passive DoF without detaching the subject from the exoskeleton. These fixations

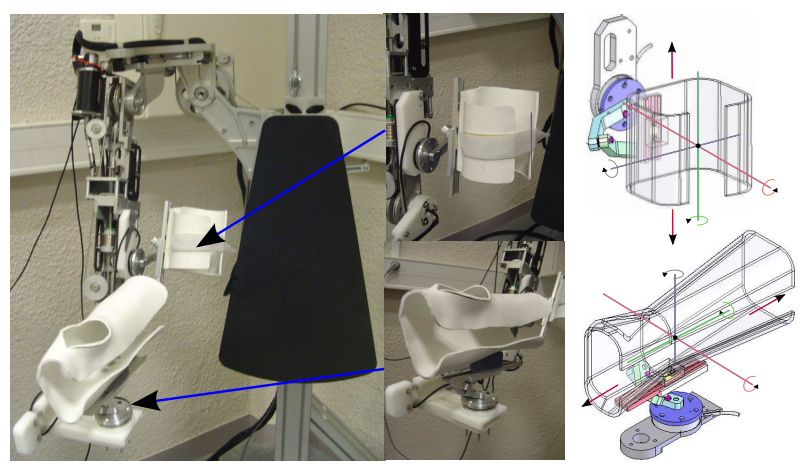

Fig. 10. The two fixations on the exoskeleton

were mounted on the 4 DoF ABLE exoskeleton. Arm fixation is placed near the elbow, just under the triceps. Forearm fixation is placed near the wrist. Thermoformable materials were also used to create two splints perfectly adapted to human morphology. These splints are serially connected to the last fixation body. Wrist splint was specifically created to lock the wrist flexions which are not studied here. Only passive pronosupination is allowed.

\section{EXPERIMENTAL RESULTS}

\section{A. Experimental setup}

An articulated mannequin was used for the experiment. Its arms possess 5 passive DoFs (a ball joint shoulder, a pivot elbow and a pronosupination). Analyzing the interaction force and torque variations at the interfaces during the same movement with isostatic fixations and without (locked case) will allow us to evaluate their impact on preventing the appearance of uncontrolled forces but also to quantify them roughly.

The mannequin was thus placed in the exoskeleton and attached through the fwo fixations. The thermoformable splints allow to avoid any looseness in the fixation and increase the contact stiffness (no foam needed).

During the experiments, the exoskeleton imposes a controlled trajectory, with a constant speed, to the mannequin arm. The experiment consists in a serie of six simple point-to-point movements (with a limited range of motion) to the same target but reached with different joints movements (thanks 


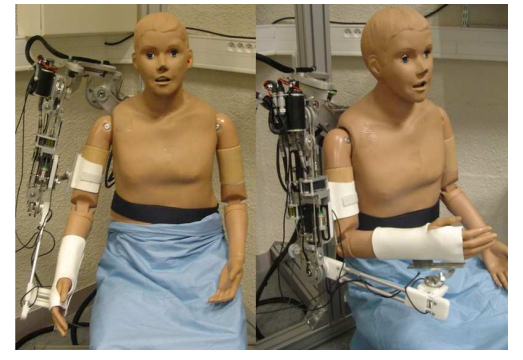

Fig. 11. Mannequin connected to the ABLE exoskeleton

to arm redundancy). Target was reached at constant and low speed $(0.05 \mathrm{~m} / \mathrm{s})$ to limit inertial forces. Due to the rigidity of the mannequin surface, the movement amplitude on every exoskeleton joint was limited to a $15^{\circ}$ maximum range of motion in order not to destroy force sensors whose maximum allowable value is about $36 \mathrm{~N}$. Indeed when exoskeleton is connected to human limb, thanks to skin and muscles deformations, the hyperstatic force level applied on the human kinematic structure (the bones) is reduced.

The use of a mannequin controlled by exoskeleton allows to obtain a perfect repeatability during the experiments. This is really representative of co-manipulation cases where the robot generates a controlled motion by applying forces, as during robotic rehabilitation or movement assistance for impaired people.

\section{B. Results and discussions}

Principal results are presented below. In Fig. 12, we plotted the undesired force absolute value and mean moment averaged norm during the experiments, for the two sensors, averaged across the six movements (moments are computed at the rotation center of the fixation). We can observe on the arm

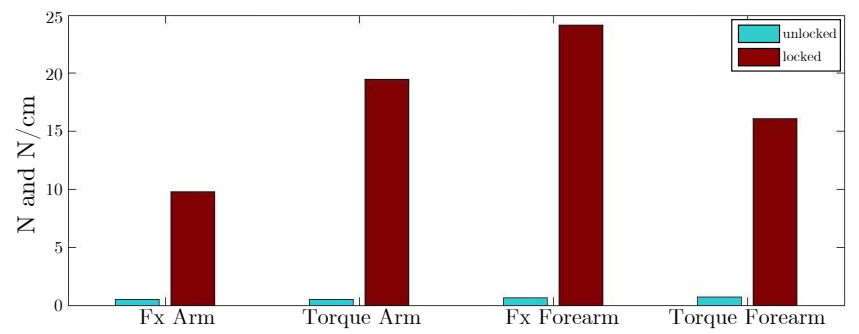

Fig. 12. Averaged absolute value of the undesired force $\left|F_{x}\right|$ and moments norm $\sqrt{\left(M_{x}^{2}+M_{y}^{2}+M_{z}^{2}\right)}$ on the two fixations (mean for the six movements)

fixation a decrease in the undesired force $\left(F_{x}\right)$ level and the undesired torques level by approximatively 95\%. For the forearm fixation, an approximative $96 \%$ decrease can be observed for the undesired force and moment components. In Fig. 13, the norm of the components $\left(F_{y}\right.$ and $\left.F_{z}\right)$ corresponding to the components transmitted by the passive fixations is presented. The exoskeleton ability to transmit forces to the subject is not altered. Note that the percentage of hyperstatic force level decrease achieved by the fixations resulting from our methodology and the obtained numerical value of the

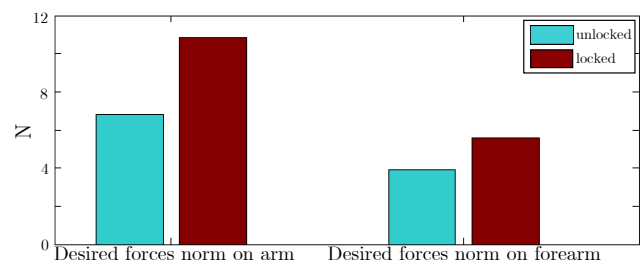

Fig. 13. Allowed forces $\left(\sqrt{\left(F_{x}^{2}+F_{y}^{2}\right)}\right)$ norm on the two fixation (mean for the six movements)

hyperstatic forces have to be interpreted. Indeed, due to the mannequin arm smallness (see Fig. 11) and it body suface rigidity, hyperstatic force level is higher than the one occuring during a comanipulation between the exoskeleton and a human subject.

\section{CONCLUSION}

In this paper we presented a methodology aimed at designing the kinematics of fixations between an exoskeleton and a human member. Thanks to this method, we realized isostatic fixations for a $4 \mathrm{DoF}$ exoskeleton and experimentally verified their benefit on minimizing uncontrollable hyperstatic forces at the human robot interface and thus on a fine control of the interaction forces. These results show that the provided solution avoids hyperstaticity but also adapts to large variations of the human limb geometry without requiring a complex adaptable robot structure.

\section{APPENDIX}

\section{A. Demonstration of Proposition 1}

1) Conditions (3) are sufficient: $[(3) \Rightarrow(2)]$.

We here suppose that conditions (3) are verified.

Because in $\mathbf{S}_{n}, \mathscr{R}_{i-1}$ is connected on one side to $\mathscr{R}_{0}$ through $\mathbf{S}_{i-1}$ and on the other side to $\mathscr{R}_{i}$ through $\mathbf{R}_{i}$ (see Fig. 3 ), one has:

$$
\forall i \in\{1 \ldots n\}, \quad{ }^{\mathbf{S}_{n}} T_{i-1}={ }^{\mathbf{S}_{i-1}} T_{i-1} \cap\left[T_{\mathbf{R}_{i}}+{ }^{\mathbf{S}_{n}} T_{i}\right],
$$

which is a recursive relationship for ${ }^{S_{n}} T_{i}$. Recalling that, by assumption, ${ }^{\mathbf{S}_{n}} T_{\mathbf{S}_{n}}=\{0\}$ (condition $3 \mathrm{c}$ ) and $T_{\mathbf{S}_{i-1}} \cap T_{\mathbf{R}_{i}}=\{0\}$ (condition $3 \mathrm{~b}$ ), this recursive law trivially leads to (2a).

Furthermore, the kinemato-static duality principle applied to the loop $\left(\mathscr{R}_{0} \rightarrow \mathscr{R}_{i-1} \rightarrow \mathscr{R}_{i} \rightarrow \mathscr{R}_{0}\right)$ in Fig. 3 writes:

$$
\forall i \in\{1 \ldots n\}, \quad \operatorname{dim}\left(\mathbf{S}_{i} W_{\mathbf{L}_{i} \rightarrow 0}\right)+\operatorname{dim}\left(T_{\mathbf{S}_{i-1}}+T_{\mathbf{R}_{i}}+T_{\mathbf{L}_{i}}\right)=6 .
$$

Thanks to condition (3a), this leads to:

$$
\forall i \in\{1 \ldots n\}, \quad{ }^{\mathbf{S}_{i}} W_{\mathbf{L}_{i} \rightarrow 0}=\{0\} .
$$

Considering again the system $\mathbf{S}_{i}$ depicted in Fig. 3, and recalling that $\mathbf{L}_{i}$ and $\mathbf{R}_{i}$ are serial chains, one has, $\forall i \in\{1 \ldots n\}$ :

$$
{ }^{\mathbf{S}_{i}} W_{\mathbf{L}_{i} \rightarrow 0}={ }^{\mathbf{S}_{i}} W_{\mathbf{L}_{i} \rightarrow i}={ }^{i} W_{\mathbf{R}_{i} \rightarrow i}={ }^{\mathbf{S}_{i}} W_{\mathbf{R}_{i} \rightarrow i-1}=\{0\} .
$$

Therefore, statically speaking, the multi-loop system $\mathbf{S}_{i-1}$ is in the same state when included in $\mathbf{S}_{i}$ than when isolated from the rest of the mechanism.

$$
\forall i \in\{2 \ldots n\}, \quad{ }^{\mathbf{S}_{i}} W_{\mathbf{L}_{i-1} \rightarrow 0}={ }^{\mathbf{S}_{i-1}} W_{\mathbf{L}_{i-1} \rightarrow 0},
$$


which, together with (13) recursively leads to condition (2b).

2) Conditions (3) are necessary : $[\overline{(3)} \Rightarrow \overline{(2)}]$.

Firstly, if condition (3c) is not verified, then ${ }^{\mathbf{S}_{n}} T_{n}=T_{\mathbf{S}_{n}} \neq\{0\}$. In this case, (2a) is not satisfied.

Secondly, if (3b) is not verified, then $\exists i,\left(T_{\mathbf{R}_{i}} \cap T_{\mathbf{S}_{i-1}}\right) \neq\{0\}$. Thanks to Eq. (11), this leads to:

$$
\exists i \in\{1 \cdots n\}, \quad{ }^{\mathbf{S}_{n}} T_{i-1} \neq\{0\},
$$

which directly contradicts (2a).

Thirdly, if (3a) is not verified, i.e.:

$$
\exists i, \operatorname{dim}\left(T_{\mathbf{S}_{i-1}}+T_{\mathbf{R}_{i}}+T_{\mathbf{L}_{i}}\right) \leq 6,
$$

then $\exists i, \quad \mathbf{S}_{i} W_{\mathbf{L}_{i \rightarrow 0}} \neq\{0\}$, meaning that $\mathbf{S}_{i}$ taken isolate is hyperstatic. Obviously, adding the rest of the mechanism to build $\mathbf{S}_{n}$, which consists of adding a parallel branch to $\mathbf{S}_{i}$ between $\mathscr{R}_{0}$ and $\mathscr{R}_{i}$ will not decrease the degree of hyperstaticity. Therefore $\exists i, \mathbf{S}_{n} W_{\mathbf{L}_{i \rightarrow 0}} \neq\{0\}$, which contradicts condition $(2 b)$.

\section{B. Singularity analysis for ABLE and the two proposed} fixation mechanisms

We study the mechanism depicted in Fig. 14: $\mathbf{R}_{1}$ is a ball joint which center is $M_{1} ; \mathbf{L}_{1}$ is composed of a ball joint which center is $P_{1}$ (with $\overrightarrow{M_{1} P_{1}}=l_{1} \cdot \overrightarrow{z_{1}}$ and $l_{1} \neq 0$ ) and a slide along $\left(P_{1}, \overrightarrow{z_{\text {arm }}}\right) ; \mathbf{R}_{2}$ is a pivot joint which axis is $\left(M_{2}, \overrightarrow{x_{2}}\right) ; \mathbf{L}_{2}$ is composed of a ball joint which center is $P_{2}$ (with $\overrightarrow{M_{2} P_{2}}=l_{2} \cdot \overrightarrow{z_{2}}$ and $\left.l_{2} \neq 0\right)$ and a slide along $\left(P_{2}, \overrightarrow{z_{\text {forearm }}}\right)$.

In order to find the singular configurations of this system, we use the necessary and sufficient conditions (3).

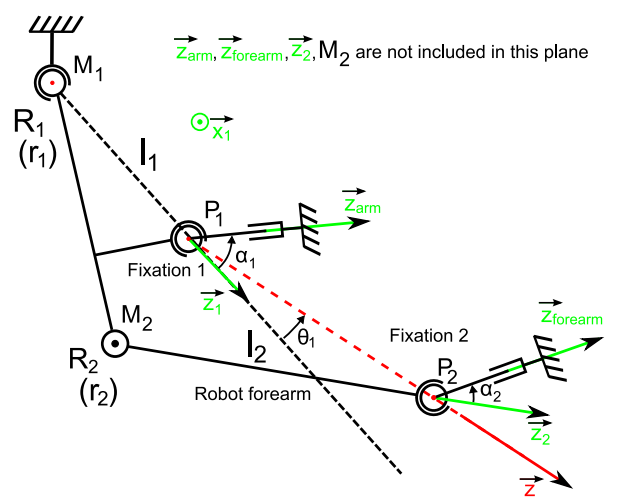

Fig. 14. Kinematics of ABLE + its fixations. The plane of the figure, perpendicular to $\vec{x}_{1}$, is defined by $M_{1}, P_{1}$ and $P_{2}$ while $M_{2}$ is off the plane.

\section{1) Examination of Condition (3a)}

- For $i=1$, (3a) writes $\operatorname{dim}\left(T_{\mathbf{R}_{1}}+T_{\mathbf{L}_{1}}\right)=6$.

At point $P_{1}$, velocities allowed by $\mathbf{L}_{1}$ belong to the vector subspace $T_{\mathbf{L}_{1}}=\operatorname{span}\left\{t_{1}, t_{2}, t_{3}, t_{4}\right\}$ and the velocities allowed by $\mathbf{R}_{1}$ belong to $T_{\mathbf{R}_{1}}=\operatorname{span}\left\{t_{5}, t_{6}, t_{3}\right\}$, with

$$
\begin{aligned}
& t_{1}=\left(x_{1}{ }^{\mathrm{T}} 0_{3}{ }^{\mathrm{T}}\right)^{\mathrm{T}}, \quad t_{3}=\left(z_{1}{ }^{\mathrm{T}} 0_{3}{ }^{\mathrm{T}}\right)^{\mathrm{T}}, \quad t_{5}=\left(x_{1}{ }^{\mathrm{T}}-l_{1} \cdot y_{1}{ }^{\mathrm{T}}\right)^{\mathrm{T}} \\
& t_{2}=\left(y_{1}{ }^{\mathrm{T}} 0_{3}{ }^{\mathrm{T}}\right)^{\mathrm{T}}, \quad t_{4}=\left(0_{3}{ }^{\mathrm{T}} z_{\text {arm }}{ }^{\mathrm{T}}\right)^{\mathrm{T}}, \quad t_{6}=\left(y_{1}{ }^{\mathrm{T}} l_{1} \cdot x_{1}{ }^{\mathrm{T}}\right)^{\mathrm{T}}
\end{aligned}
$$

Thus $T_{\mathbf{R}_{1}}+T_{\mathbf{L}_{1}}=\operatorname{span}\left\{t_{1}, \ldots, t_{6}\right\}$. Defining

$t_{5}^{\prime}=\frac{\left(t_{6}-t_{2}\right)}{l_{1}}=\left(0_{3}{ }^{\mathrm{T}} x_{1}{ }^{\mathrm{T}}\right)^{\mathrm{T}}$ and $t_{6}^{\prime}=\frac{\left(t_{1}-t_{5}\right)}{l_{1}}=\left(0_{3}{ }^{\mathrm{T}} y_{1}{ }^{\mathrm{T}}\right)^{\mathrm{T}}$, we can easily show that

$$
\left[\begin{array}{llllll}
t_{1} & t_{2} & t_{3} & t_{4} & t_{5}^{\prime} & t_{6}^{\prime}
\end{array}\right]=A\left[\begin{array}{llllll}
t_{1} & t_{2} & t_{3} & t_{4} & t_{5} & t_{6}
\end{array}\right]
$$

with $\operatorname{det}(A)=\frac{1}{l_{1}^{2}}$. Since $l_{1} \neq 0, \tau_{1}=\left\{t_{1}, . ., t_{6}\right\}$ is a basis of $\mathbb{R}^{6}$ if and only if $\tau_{2}=\left\{t_{1}, . ., t_{4}, t_{5}^{\prime}, t_{6}^{\prime}\right\}$ is a basis of $\mathbb{R}^{6}$. Let's consider now $a_{i} \in \mathbb{R}, i \in\{1, . ., 6\}$ such that:

$$
a_{1} t_{1}+a_{2} t_{2}+a_{3} t_{3}+a_{4} t_{4}+a_{5} t_{5}^{\prime}+a_{6} t_{6}^{\prime}=0
$$

It is trivial to show that $a_{1}=a_{2}=a_{3}=0, a_{4} d_{z}=0, a_{6}+$ $a_{4} d_{y}=0$ and $a_{5}+a_{4} d_{x}=0$ where $\overrightarrow{z_{a r m}}=d_{x} \overrightarrow{x_{1}}+d_{y} \overrightarrow{y_{1}}+d_{z} \overrightarrow{z_{1}}$. If $d_{z} \neq 0$ then $a_{6}=a_{5}=a_{4}=0$. Therefore, $\tau_{2}$ and $\tau_{1}$ are bases of $\mathbb{R}^{6}$. Otherwise, there exists a non null combination of $a_{i}$ that verifies (17) which means that $\tau_{2}$ and $\tau_{1}$ are not free anymore. Condition (3a) is thus verified for $i=1$ if and only if $\overrightarrow{z_{\text {arm }}} \cdot \overrightarrow{z_{1}} \neq 0$. This is equivalent to $\alpha_{1} \neq \pm \frac{\pi}{2}$ and this is a singular value to be avoided. In the rest of the study we will thus consider that $\overrightarrow{z_{\text {arm }}} \cdot \overrightarrow{z_{1}} \neq 0$.

- For $i=2$, (3a) writes $\operatorname{dim}\left(T_{\mathbf{S}_{1}}+T_{\mathbf{R}_{2}}+T_{\mathbf{L}_{2}}\right)=6$.

We know that $T_{\mathbf{S}_{1}}=T_{\mathbf{R}_{1}} \cap T_{\mathbf{L}_{1}}$. Let's consider $t \in T_{\mathbf{L}_{1}}$ and $t^{\prime} \in$ $T_{\mathbf{R}_{1}}$. One has:

$$
\begin{aligned}
& \exists\left(\alpha_{1}, \alpha_{2}, \alpha_{3}, \alpha_{4}\right) \quad \text { such that } t=\sum_{i=1}^{4} \alpha_{i} t_{i} \\
& \exists\left(\alpha_{1}^{\prime}, \alpha_{2}^{\prime}, \alpha_{3}^{\prime},\right) \quad \text { such that } t^{\prime}=\alpha_{1}^{\prime} t_{5}+\alpha_{2}^{\prime} t_{6}+\alpha_{3}^{\prime} t
\end{aligned}
$$

Using $\overrightarrow{z_{a r m}} \cdot \overrightarrow{z_{1}} \neq 0$, one easily gets:

$$
t=t^{\prime} \Leftrightarrow \alpha_{1}=\alpha_{2}=\alpha_{4}=\alpha_{1}^{\prime}=\alpha_{2}^{\prime}=0 .
$$

or:

$$
t=t^{\prime} \Leftrightarrow t=\alpha_{3} t_{3}=\alpha_{3}^{\prime} t_{3}
$$

In other words, at point $P_{1}$ :

$$
T_{\mathbf{S}_{1}}=T_{\mathbf{R}_{1}} \cap T_{\mathbf{L}_{1}}=\operatorname{span}\left(\left\{t_{3}\right\}\right)=\operatorname{span}\left(\left\{\left(z_{1}{ }^{\mathrm{T}} 0_{3}{ }^{\mathrm{T}}\right)^{\mathrm{T}}\right\}\right) .
$$

We know write twists at point $P_{2}$. We get: $T_{\mathbf{S}_{1}}=\operatorname{span}\left(\left\{t_{7}\right\}\right)$, $T_{\mathbf{R}_{2}}=\operatorname{span}\left(\left\{t_{8}\right\}\right)$ and $T_{\mathbf{L}_{2}}=\operatorname{span}\left(\left\{\begin{array}{llll}t_{9} & t_{10} & t_{11} & t_{12}\end{array}\right\}\right)$, with:

$$
\begin{array}{r}
t_{7}=\left(z_{1}{ }^{\mathrm{T}} l \sin \theta_{1} x_{1}{ }^{\mathrm{T}}\right)^{\mathrm{T}}, \quad t_{8}=\left(x_{2}{ }^{\mathrm{T}}-l_{2} y_{2}{ }^{\mathrm{T}}\right)^{\mathrm{T}}, \quad t_{9}=\left(x_{2}{ }^{\mathrm{T}} 0^{\mathrm{T}}\right)^{\mathrm{T}} \\
t_{10}=\left(y_{2}{ }^{\mathrm{T}} 0^{\mathrm{T}}\right)^{\mathrm{T}}, \quad t_{11}=\left(z_{2}{ }^{\mathrm{T}} 0^{\mathrm{T}}\right)^{\mathrm{T}}, \quad t_{12}=\left(0^{\mathrm{T}} z_{\text {forearm }}{ }^{\mathrm{T}}\right)^{\mathrm{T}},
\end{array}
$$

where $\overrightarrow{P_{1} P_{2}}=: l \vec{z}$ and $\theta_{1}:=\left(\overrightarrow{\overrightarrow{z_{1}}, \vec{z}}\right)$ measured around $\vec{x}_{1}$. Thus $T_{\mathbf{S}_{1}}+T_{\mathbf{R}_{2}}+T_{\mathbf{L}_{2}}=\operatorname{span}\left(\left\{t_{7}, t_{8}, t_{9}, t_{10}, t_{11}, t_{12}\right\}\right)$.

Suppose first that $\sin \theta_{1}=0$. Then, denoting $\overrightarrow{z_{1}}=z_{1 x} \cdot \overrightarrow{x_{2}}+$ $z_{1 y} \cdot \overrightarrow{y_{2}}+z_{1 z} \cdot \overrightarrow{z_{2}}$, one gets:

$$
t_{7}=z_{1 x} t_{9}+z_{1 y} t_{10}+z_{1 z} t_{12}
$$

In this particular case, $\left\{t_{7} \ldots t_{12}\right\}$ is not a basis, which identifies a second singular configuration, when $M_{1}, P_{1}$ and $P_{2}$ are aligned. In the rest of the study we will thus assume that this 
singular configuration is also avoided, that is: $\sin \theta_{1} \neq 0$. Defining

$$
\begin{aligned}
& t_{7}^{\prime}=\frac{\left(t_{7}-z_{1 x} t_{9}-z_{1 y} t_{10}-z_{1 z} t_{12}\right)}{l \sin \theta_{1}}=\left(0^{\mathrm{T}} x_{1}{ }^{\mathrm{T}}\right)^{\mathrm{T}}, \text { and } \\
& t_{8}^{\prime}=\frac{\left(t_{10}-t_{8}\right)}{l_{2}}=\left(0^{\mathrm{T}} y_{2}^{\mathrm{T}}\right)^{\mathrm{T}},
\end{aligned}
$$

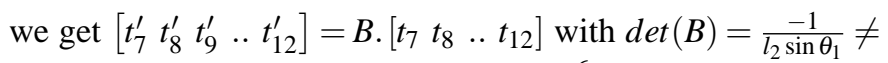
0 . Thus $\tau_{3}=\left\{t_{7} \ldots t_{12}\right\}$ is a basis of $\mathbb{R}^{6}$ if and only if $\tau_{4}=$ $\left\{t_{7}^{\prime} \ldots t_{12}^{\prime}\right\}$ is a basis of $\mathbb{R}^{6}$. Lets's consider $b_{i} \in \mathbb{R}, i \in\{1, . ., 6\}$ such that:

$$
b_{1} t_{7}^{\prime}+b_{2} t_{8}^{\prime}+b_{3} t_{9}+b_{4} t_{10}+b_{5} t_{11}+b_{6} t_{12}=0 .
$$

It comes easily that $b_{3}=b_{4}=b_{5}=0$ and $b_{1} t_{7}^{\prime}+b_{2} t_{8}^{\prime}+b_{6} t_{12}^{\prime}=$ 0 which is equivalent to $b_{1} \overrightarrow{x_{1}}+b_{2} \overrightarrow{y_{2}}+b_{6} \overrightarrow{z_{\text {forearm }}}=\overrightarrow{0}$. The necessary and sufficient conditions to have a non-null triplet $b_{1}, b_{2}, b_{6}$ verifying the previous equation is that $\overrightarrow{x_{1}}, \overrightarrow{y_{2}}, \overrightarrow{z_{\text {forearm }}}$ are coplanar. This identifies a third singularity, which, again, is supposed to be avoided in the rest of the study.

2) Examination of the condition (3b)

- For $i=1$, since $T_{\mathbf{S}_{0}}=\{0\}$, one directly gets $\operatorname{dim}\left(T_{\mathbf{S}_{0}} \cap\right.$ $\left.T_{\mathbf{L}_{1}}\right)=0$.

- For $i=2$, it is necessary to verify that $\operatorname{dim}\left(T_{\mathbf{S}_{1}} \cap T_{\mathbf{L}_{2}}\right)=0$. Let's consider $t \in T_{\mathbf{S}_{1}}$ and $t^{\prime} \in T_{\mathbf{L}_{2}}$. One has:

$$
\begin{aligned}
\exists \alpha_{1} & \in \mathbb{R} \quad / \quad t=\alpha_{1} t_{7} \\
\exists \alpha_{1}^{\prime}, \alpha_{2}^{\prime}, \alpha_{3}^{\prime}, \alpha_{4}^{\prime} & \in \mathbb{R} \quad / \quad t^{\prime}=\alpha_{1}^{\prime} t_{9}+\alpha_{2}^{\prime} t_{10}+\alpha_{3}^{\prime} t_{11}+\alpha_{4}^{\prime} t_{12} .
\end{aligned}
$$

One easily shows that $t=t^{\prime}$ is equivalent to:

$$
\left\{\begin{array}{l}
\alpha_{1} l \sin \theta_{1} \overrightarrow{x_{1}}+\alpha_{4}^{\prime} \overrightarrow{z_{\text {forearm }}}=\overrightarrow{0} \\
\left(\alpha_{1} z_{1 x}+\alpha_{1}^{\prime}\right) \overrightarrow{x_{2}}+\left(\alpha_{1} z_{1 y}+\alpha_{2}^{\prime}\right) \overrightarrow{y_{2}}+\left(\alpha_{1} z_{1 z}+\alpha_{3}^{\prime}\right) \overrightarrow{z_{2}}=\overrightarrow{0}
\end{array}\right.
$$

Since $\overrightarrow{x_{1}}$ is not colinear to $\overrightarrow{z_{\text {forearm }}}$, the first equation leads to $\alpha_{1}=\alpha_{4}^{\prime}=0$. Similarly, since $\left\{\overrightarrow{x_{2}}, \overrightarrow{y_{2}}, \overrightarrow{z_{2}}\right\}$ forms a basis, $\alpha_{1}^{\prime}=\alpha_{2}^{\prime}=\alpha_{3}^{\prime}=0$. In conclusion, $\operatorname{dim}\left(T_{\mathbf{S}_{1}} \cap T_{\mathbf{L}_{2}}\right)=\{0\}$.

3) Examination of the condition (3c)

For the considered example, $n=2$ and condition (3c) writes $\operatorname{dim}\left(T_{\mathbf{S}_{2}}\right)=0$. Since $T_{\mathbf{S}_{2}}=\left(T_{\mathbf{S}_{1}}+T_{\mathbf{R}_{2}}\right) \cap T_{\mathbf{L}_{2}}$, we need to verify that any vector that belongs to both $\left(T_{\mathbf{S}_{1}}+T_{\mathbf{R}_{2}}\right)$ and $T_{\mathbf{L}_{2}}$ is null. Let's consider $t \in\left(T_{\mathbf{S}_{1}}+T_{\mathbf{R}_{2}}\right)$ and $t^{\prime} \in T_{\mathbf{L}_{2}}$. One has:

$$
\begin{aligned}
\exists \alpha_{1}, \alpha_{2} \in \mathbb{R} \quad / \quad t=\alpha_{1} t_{7}+\alpha_{2} t_{8} \\
\exists \alpha_{1}^{\prime}, . ., \alpha_{4}^{\prime} \in \mathbb{R} \quad / \quad t^{\prime}=\alpha_{1}^{\prime} t_{9}+\alpha_{2}^{\prime} t_{1} 0+\alpha_{3}^{\prime} t_{1} 1+\alpha_{4}^{\prime} t_{1} 2
\end{aligned}
$$

Therefore $t=t^{\prime}$ is equivalent to:

$$
\left\{\begin{array}{l}
\alpha_{1} l \sin \theta_{1} \overrightarrow{x_{1}}-\alpha_{2} l_{2} \overrightarrow{y_{2}}+\alpha_{4}^{\prime} \overrightarrow{z_{\text {forearm }}}=\overrightarrow{0} \\
\left(\alpha_{1} z_{1 x}+\alpha_{1}^{\prime}+\alpha_{2}\right) \overrightarrow{x_{2}}+\left(\alpha_{1} z_{1 y}+\alpha_{2}^{\prime}\right) \overrightarrow{y_{2}}+\left(\alpha_{1} z_{1 z}+\alpha_{3}^{\prime}\right) \overrightarrow{z_{2}}=\overrightarrow{0}
\end{array}\right.
$$

The first of these two equations leads to $\alpha_{1}=\alpha_{2}=\alpha_{4}^{\prime}=0$ since it is supposed that $\overrightarrow{x_{1}}, \overrightarrow{y_{2}}$ and $\overrightarrow{z_{\text {forearm }}}$ are not coplanar in order to avoid the third singularity, and $\sin \theta_{1} \neq 0$ in order to avoid the second singularity. Therefore, the second equation leads to $\alpha_{1}=\alpha_{2}=\alpha_{4}^{\prime}=0$ because $\left\{\overrightarrow{x_{2}}, \overrightarrow{y_{2}}, \overrightarrow{z_{2}}\right\}$ forms a basis. In conclusion, $t=t^{\prime} \Rightarrow t=0$, or $\operatorname{dim}\left(T_{\mathbf{S}_{2}}\right)=0$.

4) Summary.

In conclusion, we identified three singularities:
1) $\overrightarrow{z_{\text {arm }}} \cdot \overrightarrow{z_{1}}=0$ representing the case where the passive slide, mounted parallel to the upper arm axis, is perpendicular to the robot upper limb axis. This case will never appear since the angle between $\overrightarrow{z_{a r m}}$ and $\overrightarrow{z_{1}}$ reflects small discrepancies between the exoskeleton and human kinematics, and remains smaller than a few degrees.

2) $\sin \theta_{1}=0$ representing the case where $M_{1}, P_{1}$ and $P_{2}$ are aligned. This singular configuration can be avoided by limiting the range of motion for the robot elbow to a few degrees before full extension.

3) $\overrightarrow{x_{1}}, \overrightarrow{y_{2}}$ and $\overrightarrow{z_{\text {forearm }}}$ coplanar. This configuration does not appear in practice, since in the nominal configuration, $\overrightarrow{x_{1}}$ is perpendicular to the plane generated by $\overrightarrow{y_{2}}$ and $\overrightarrow{z_{\text {forearm }}}$.

Therefore, under normal conditions of operation, the ABLE exoskeleton with its two fixations never falls into a singular configuration.

\section{REFERENCES}

[1] A.B. Zoss, H. Kazerooni, and A. Chu. Biomechanical design of the berkeley lower extremity exoskeleton (bleex). Mechatronics, IEEE/ASME Transactions on, 11(2):128 -138, april 2006.

[2] M. Mihelj, T. Nef, and R. Riener. Armin ii - 7 dof rehabilitation robot: mechanics and kinematics. In Robotics and Automation, 2007 IEEE International Conference on, pages 4120 -4125, 10-14 2007.

[3] J.C. Perry, J. Rosen, and S. Burns. Upper-limb powered exoskeleton design. Mechatronics, IEEE/ASME Transactions on, 12(4):408 -417, aug. 2007.

[4] Jos L. Pons. Wearable Robots: Biomechatronic Exoskeletons. Wiley, April 2008.

[5] Stephen H. Scott and David A. Winter. Biomechanical model of the human foot: Kinematics and kinetics during the stance phase of walking. Journal of Biomechanics, 26(9):1091-1104, September 1993.

[6] F.C.T. Van der Helm, H.E.J. Veeger, G.M. Pronk, L.H.V. Van der Woude, and R.H. Rozendal. Geometry parameters for musculoskeletal modelling of the shoulder system. Journal of Biomechanics, 25(2):129144, February 1992.

[7] A. Schiele. An explicit model to predict and interpret constraint force creation in phri with exoskeletons. In Robotics and Automation, 2008. ICRA 2008. IEEE International Conference on, pages 1324 -1330, 19232008 .

[8] A. Schiele and F.C.T. van der Helm. Kinematic design to improve ergonomics in human machine interaction. Neural Systems and Rehabilitation Engineering, IEEE Transactions on, 14(4):456 -469, dec. 2006.

[9] L W Lamoreux. Kinematic measurements in the study of human walking. Bull Prosthet Res., 10(15):3-84, 1971. PMID: 5131748.

[10] KL Markolf, JS Mensch, and HC Amstutz. Stiffness and laxity of the knee-the contributions of the supporting structures. a quantitative in vitro study. J Bone Joint Surg Am, 58(5):583-594, 1976.

[11] C. Diez-Martnez, J. Rico, J. Cervantes-Snchez, and J. Gallardo. Mobility and connectivity in multiloop linkages. In Advances in Robot Kinematics, pages 455-464. 2006.

[12] M. Stokdijk, C. G. M. Meskers, H. E. J. Veeger, Y. A. de Boer, and P. M. Rozing. Determination of the optimal elbow axis for evaluation of placement of prostheses. Clinical Biomechanics, 14(3):177-184, March 1999.

[13] K. J. Waldron. The constraint analysis of mechanisms. Journal of Mechanisms, 1(2):101-114, 1966.

[14] P. Garrec, J.P. Friconneau, Y. Measson, and Y. Perrot. Able, an innovative transparent exoskeleton for the upper-limb. Intelligent Robots and Systems, 2008. IROS 2008. IEEE/RSJ International Conference on, pages $1483-1488$, Sept. 2008.

[15] Garrec. P. French patent: Transmission vis, ecrou et cable attache a la vis - fr0101630, 2000 (eur 01938347.0-2421 and us 10/296,740 (screw and nut transmission and cable). 2000. 